https://doi.org/10.15407/ujpe66.10.833

A. ARSLANALIEV,${ }^{1,2}$ Y. KOSTYLENKO ${ }^{1}$ O. SHEBEKO $^{1}$

${ }^{1}$ Akhiezer Institute for Theoretical Physics,

National Science Center "Kharkiv Institute of Physics and Technology"

(1, Akademichna Str., Kharkiv 61108, Ukraine)

${ }^{2}$ V.N. Karazin Kharkiv National University

(4, Svobody Sq., Kharkiv 61022, Ukraine; e-mail: arslanaliev.kh@gmail.com)

\title{
A NEW FAMILY OF INTERACTIONS BETWEEN CLOTHED PARTICLES IN QED
}

\begin{abstract}
The method of unitary clothing transformations (UCTs) has been applied to the quantum electrodynamics (QED) by using the clothed particle representation (CPR). Within $C P R$, the Hamiltonian for interacting electromagnetic and electron-positron fields takes the form in which the interaction operators responsible for such two-particle processes as $e^{-} e^{-} \rightarrow e^{-} e^{-}$, $e^{+} e^{+} \rightarrow e^{+} e^{+}, e^{-} e^{+} \rightarrow e^{-} e^{+}, e^{-} e^{+} \rightarrow \gamma \gamma, \gamma \gamma \rightarrow e^{-} e^{+}, \gamma e^{-} \rightarrow \gamma e^{-}$, and $\gamma e^{+} \rightarrow \gamma e^{+}$are obtained on the same physical footing. These novel interactions include the off-energy-shell and recoil effects (the latter without any expansion in $(v / c)^{2}$-series) and their on-energyshell matrix elements reproduce the well-known results derived within the perturbation theory based on the Dyson expansion for the S-matrix (in particular, the Møller formula for the $e^{-} e^{-}$-scattering, the Bhabha formula for $e^{-} e^{+}$-scattering, and the Klein-Nishina one for the Compton scattering).
\end{abstract}

Keywords: unitary clothing transformations, quantum electrodynamics, clothed particles, off-energy-shell effects.

\section{Introduction}

Starting from the instant form of relativistic quantum dynamics for a system of interacting particles, where, amongst the ten generators of the Poincaré group $\Pi$, only the Hamiltonian $H$ and the boost operator $\boldsymbol{B}$ carry interactions, we have built up the Hamiltonian in CPR for the interacting electron-positron and photon fields. In this connection, let as recall that the transition from the primary "bare" particle representation (BPR) with its bare particle states to CPR is implemented via the UCT method put forward by Greenberg and Schweber [1] and developed in Refs. [2-5].

Currently, the UCT method has been applied in the mesodynamics to the processes that involve interac-

(C) A. ARSLANALIEV, Y. KOSTYLENKO, O. SHEBEKO, 2021

ISSN 2071-0194. Ukr. J. Phys. 2021. Vol. 66, No. 10 tions of the clothed $\pi, \eta, \rho, \omega, \delta, \sigma$ mesons and nucleons $[3,5-9]$. The applications of this method in QED and quantum chromodynamics (QCD) are shown in [10-13]. In the present work, we will show explicit expressions for a new family of interactions between the clothed particles in QED.

The outline of this paper is as follows. In the next section, we will take a look briefly at the underlying formalism and structure of a Hamiltonian in CPR. This is followed by a section that shows exactly how the QED Hamiltonian breaks down into a set of interactions and provides a derivation of one of them as an example. Then we will present the explicit expressions for all the interaction operators included in the Hamiltonian in the $e^{2}$-order.

\section{Underlying Formalism}

The method in question is aimed at expressing a field Hamiltonian through the so-called clothed-particle 
creation (annihilation) operators $\alpha_{c}$, e.g., $c_{c}^{\dagger}\left(c_{c}\right)$, $b_{c}^{\dagger}\left(b_{c}\right)$, and $d_{c}^{\dagger}\left(d_{c}\right)$ via UCTs $W\left(\alpha_{c}\right)=W(\alpha)=\exp R$, $R=-R^{\dagger}$ in the similarity transformation

$\alpha=W\left(\alpha_{c}\right) \alpha_{c} W^{\dagger}\left(\alpha_{c}\right)$

that connects a primary set $\alpha$ in BPR with the new operators in CPR.

A key point of the clothing procedure is to remove the so-called bad terms from the Hamiltonian

$H \equiv H(\alpha)=H_{F}(\alpha)+H_{I}(\alpha)=$

$=W\left(\alpha_{c}\right) H\left(\alpha_{c}\right) W^{\dagger}\left(\alpha_{c}\right) \equiv K\left(\alpha_{c}\right)$.

By definition, such terms prevent the physical vacuum $|\Omega\rangle$ (the $H$ lowest energy eigenstate) and the one-clothed-particle states $|n\rangle_{c}=a_{c}^{\dagger}(n)|\Omega\rangle$ to be $H$ eigenvectors for all $n$ included. The bad terms occur every time, when any normally ordered product

$a^{\dagger}\left(1^{\prime}\right) a^{\dagger}\left(2^{\prime}\right) \ldots a^{\dagger}\left(n_{C}^{\prime}\right) a\left(n_{A}\right) \ldots a(2) a(1)$

of the class $[$ C.A] embodies at least one substructure $\in[k .0](k=1,2, \ldots)$ or $/$ and $[k .1](k=2,3, \ldots)$. In this context, all primary Yukawa-type (trilinear) couplings should be eliminated from the interaction $V(\alpha)$ that enters

$H_{I}(\alpha)=V(\alpha)+$ mass and vertex counterterms.

It results in the form

$H=K_{F}\left(\alpha_{c}\right)+K_{I}\left(\alpha_{c}\right)=K$,

where the free part $K_{F}\left(\alpha_{c}\right)=H_{F}\left(\alpha_{c}\right)$, while the operator $K_{I}\left(\alpha_{c}\right)$ contains interactions between the clothed particles. By construction, the latter has the property

$K_{I}\left(\alpha_{c}\right)|\Omega\rangle=K_{I}\left(\alpha_{c}\right)|n\rangle_{c} \equiv 0$.

For a boson-fermion (meson-nucleon, photon-electron) system, we have the decomposition

$K_{I}\left(\alpha_{c}\right)=K(f f \rightarrow f f)+K(\bar{f} \bar{f} \rightarrow \bar{f} \bar{f})+$

$+K(f \bar{f} \rightarrow f \bar{f})+K(b f \rightarrow b f)+K(b \bar{f} \rightarrow b \bar{f})+$

$+K\left(f \bar{f} \leftrightarrow b b^{\prime}\right)+K(f f \leftrightarrow b f f)+$

$+K(f \bar{f} \leftrightarrow 3 b)+\ldots$,

where separate contributions are responsible for different physical processes. So, for instance, the operators $K(\gamma e \rightarrow \gamma e), K(e e \leftrightarrow \gamma e e)$ and $K(3 N \rightarrow 3 N)$ can be used in describing the Compton scattering on an electron, the electron-electron bremsstrahlung and model three-nucleon forces, respectively.

\section{QED Hamiltonian in CPR}

In the Coulomb gauge (used here), the interaction Hamiltonian of spinor QED is given by (cf., for example, Eqs. (8.4.3) and (8.4.23) in [14])

$V_{\mathrm{qed}}=\int d \boldsymbol{x} V_{\mathrm{qed}}(\boldsymbol{x})=\int d \boldsymbol{x} J^{\mu}(\boldsymbol{x}) A_{\mu}(\boldsymbol{x})+V_{\mathrm{Coul}}$,

with the electron-positron current density $J^{\mu}(\boldsymbol{x})=$ $=e: \bar{\psi}(\boldsymbol{x}) \gamma^{\mu} \psi(\boldsymbol{x}):$, where the colon symbol denotes the normal ordering, and the Coulomb part,

$V_{\text {Coul }}=\frac{1}{2} \int d \boldsymbol{x} \int d \boldsymbol{y} \frac{J^{0}(\boldsymbol{x}) J^{0}(\boldsymbol{y})}{4 \pi|\boldsymbol{x}-\boldsymbol{y}|} e^{-\lambda|\boldsymbol{x}-\boldsymbol{y}|}$.

Admittedly, the exponential factor with the parameter $\lambda>0$ set to zero at the end of all calculations is introduced to deal with infrared divergences. Evidently, the interaction density $V_{\text {qed }}(x)$ cannot be scalar, i.e., does not possesses the property to be invariant with respect to the Poincaré group $\Pi$, viz.,

$U_{F}(\Lambda, a) V(x) U_{F}^{-1}(\Lambda, a)=V(\Lambda x+a)$,

$\forall \Lambda \in L_{+}$and arbitrary spacetime shifts $a=\left(a^{0}, \boldsymbol{a}\right)$

The correspondence $(\Lambda, a) \rightarrow U_{F}(\Lambda, a)$ between elements $(\Lambda, a) \in \Pi$ and unitary transformations $U_{F}(\Lambda, a)$ realizes an irreducible representation of $\Pi$ in the Hilbert space of states for free (non-interacting) fields. Here, $L_{+}$is the homogeneous (proper) orthochronous Lorentz group. In this respect, for the Coulomb gauge (CG), where the photon field $A_{\mu}(\boldsymbol{x})$ is introduced in such a way to have $A_{0}(\boldsymbol{x}) \equiv 0$, we cannot use the so-called Belinfante ansatz to construct the boost generator $\boldsymbol{N}$, i.e., put, for example,

$\boldsymbol{N}_{\mathrm{qed}}=-\int \boldsymbol{x} V_{\mathrm{qed}}(\boldsymbol{x}) d \boldsymbol{x}$.

Therefore, one has to seek other ways to provide the relativistic invariance $(\mathrm{RI})$ in the Dirac sense (see, e.g., [15]).

In addition, the Fourier expansions

$\psi(\boldsymbol{x})=\sqrt{\frac{m}{(2 \pi)^{3}}} \int \frac{d \boldsymbol{p}}{E_{\boldsymbol{p}}} \sum_{\mu}(u(p \mu) b(p \mu)+$

$\left.+v\left(p_{-} \mu\right) d^{\dagger}\left(p_{-} \mu\right)\right) e^{i \boldsymbol{p} \cdot \boldsymbol{x}}$,

$A_{\mu}(\boldsymbol{x})=\frac{1}{\sqrt{2(2 \pi)^{3}}} \int \frac{d \boldsymbol{k}}{\omega_{\boldsymbol{k}}} \sum_{\sigma}\left(e_{\mu}(k \sigma) c(k \sigma)+\right.$

$\left.+e_{\mu}\left(k_{-} \sigma\right) c^{\dagger}\left(k_{-} \sigma\right)\right) e^{i \boldsymbol{k} \cdot \boldsymbol{x}}$,

ISSN 2071-0194. Ukr. J. Phys. 2021. Vol. 66, No. 10 
where

$p_{-}=\left(E_{\boldsymbol{p}},-\boldsymbol{p}\right), k_{-}=\left(\omega_{\boldsymbol{k}},-\boldsymbol{k}\right), \omega_{\boldsymbol{k}}=\sqrt{\boldsymbol{k}^{2}+\lambda^{2}}$

the photon energy, and $E_{\boldsymbol{p}}=\sqrt{\boldsymbol{p}^{2}+m^{2}}$ the electron energy, are employed to express the Hamiltonian (other generators of the Poincaré group, currents, etc.) through the creation and annihilation operators that compose the set $\alpha_{c}$.

The two independent 'polarization' vectors in Eq. (7) with the helicities $\sigma= \pm 1$, such that $\boldsymbol{k} \cdot \boldsymbol{e}(k \sigma)=0$ and $e^{0}(k \sigma)=0$, are normalized (see [14], Sect. 8.5), in terms of the timelike vector $n=(1,0,0,0)$ so

$\sum_{\sigma} e_{\mu}(k \sigma) e_{\nu}^{*}(k \sigma)=-g_{\mu \nu}+\omega_{\boldsymbol{k}} \frac{k_{\mu} n_{\nu}+k_{\nu} n_{\mu}}{\boldsymbol{k}^{2}}-\frac{k_{\mu} k_{\nu}}{\boldsymbol{k}^{2}}-$

$-\frac{k^{2}}{\boldsymbol{k}^{2}} n_{\mu} n_{\nu} \equiv P_{\mu \nu}(k)$.

Substituting these expansions into the Hamiltonian of interest, in accordance with the prescription given above, we perform the first clothing transformation $W^{(1)}=\exp \left[R^{(1)}\right]\left(R^{(1)^{\dagger}}=-R^{(1)}\right)$ which eliminates the primary interactions $V^{(1)}$ in the $e^{1}$-order assuming that $V^{(1)}$ consists of the bad terms only. It is the case of photons with the interaction in the $\mathrm{D}$ picture

$V^{(1)}(t)=\int d \boldsymbol{x} V_{\mathrm{qed}}^{(1)}(x) \equiv \int d \boldsymbol{x} J^{\mu}(x) A_{\mu}(x)$,

which is trilinear in the creation and annihilation operators involved.

The operator $R^{(1)}$ obeys the equation

$\left[R^{(1)}, H_{F}\right]+V^{(1)}=0$,

which has a solution

$R^{(1)}=-i \lim _{\varepsilon \rightarrow 0+} \int_{0}^{\infty} V_{D}^{(1)}(t) e^{-\varepsilon t} d t$,

if $m_{\gamma}<2 m$. Evidently, this inequality is valid with $m_{\gamma}=\lambda$ and $m=m_{e^{+}}=m_{e^{-}}$. One should stress that, from this moment, all the bare-particle operators $\alpha$ are replaced by the clothed-particle counterparts.

In this paper, we will confine ourselves to the consideration of the interaction operators that appear in the decomposition

$K_{I}\left(\alpha_{c}\right)=K\left(e^{-} e^{-} \rightarrow e^{-} e^{-}\right)+K\left(e^{+} e^{+} \rightarrow e^{+} e^{+}\right)+$

ISSN 2071-0194. Ukr. J. Phys. 2021. Vol. 66, No. 10

$$
\begin{aligned}
& +K\left(e^{-} e^{+} \rightarrow e^{-} e^{+}\right)+K\left(e^{-} e^{+} \rightarrow \gamma \gamma\right)+ \\
& +K\left(\gamma \gamma \rightarrow e^{-} e^{+}\right)+K\left(\gamma e^{-} \rightarrow \gamma e^{-}\right)+ \\
& +K\left(\gamma e^{+} \rightarrow \gamma e^{+}\right) .
\end{aligned}
$$

For example, in the $e^{2}$-order we find the $e^{-} e^{-}$-scattering operator

$K_{e^{-} e^{-} \rightarrow e^{-} e^{-}}^{(2)}=\frac{1}{2}\left[R^{(1)}, V^{(1)}\right]_{e^{-} e^{-} \rightarrow e^{-} e^{-}}+$

$+V_{\text {Coul } e^{-} e^{-} \rightarrow e^{-} e^{-}}$.

Here, we have

$V^{(1)}=-\frac{1}{2} \frac{m e}{\sqrt{2(2 \pi)^{3}}} \int \frac{d \boldsymbol{k}}{\omega_{\boldsymbol{k}}} \frac{d \boldsymbol{p}^{\prime}}{E_{\boldsymbol{p}^{\prime}}} \frac{d \boldsymbol{p}}{E_{\boldsymbol{p}}} \delta\left(\boldsymbol{p}^{\prime}-\boldsymbol{p}-\boldsymbol{k}\right) \times$
$\times e^{\mu}(k) \bar{u}\left(p^{\prime}\right) \gamma_{\mu} u(p) b_{c}^{\dagger}\left(p^{\prime}\right) b_{c}(p) c_{c}(k)+$ H.c.,
$R^{(1)}=-\frac{1}{2} \frac{m e}{\sqrt{2(2 \pi)^{3}}} \int \frac{d \boldsymbol{k}}{\omega_{\boldsymbol{k}}} \frac{d \boldsymbol{p}^{\prime}}{E_{\boldsymbol{p}^{\prime}}} \frac{d \boldsymbol{p}}{E_{\boldsymbol{p}}} \frac{\delta\left(\boldsymbol{p}^{\prime}-\boldsymbol{p}-\boldsymbol{k}\right)}{E_{\boldsymbol{p}^{\prime}}-E_{\boldsymbol{p}}-\omega_{\boldsymbol{k}}} \times$
$\times e^{\mu}(k) \bar{u}\left(p^{\prime}\right) \gamma_{\mu} u(p) b_{c}^{\dagger}\left(p^{\prime}\right) b_{c}(p) c_{c}(k)-$ H.c.

Henceforth, we omit the polarization indices. Now after a simple algebra with the covariant commutations

$\left\{b(p \mu), b^{\dagger}\left(p^{\prime} \mu^{\prime}\right)\right\}=\left\{d(p \mu), d^{\dagger}\left(p^{\prime} \mu^{\prime}\right)\right\}=$

$=E_{\boldsymbol{p}} \delta_{\mu \mu^{\prime}} \delta\left(\mathbf{p}-\mathbf{p}^{\prime}\right)$,

$\left[c(k \sigma), c^{\dagger}\left(k^{\prime} \sigma^{\prime}\right)\right]=\omega_{\boldsymbol{k}} \delta_{\sigma \sigma^{\prime}} \delta\left(\mathbf{k}-\mathbf{k}^{\prime}\right)$,

we get

$\frac{1}{2}\left[R^{(1)}, V^{(1)}\right]_{e^{-} e^{-} \rightarrow e^{-} e^{-}}=\int \frac{d \boldsymbol{p}_{1}^{\prime}}{E_{\boldsymbol{p}_{1}^{\prime}}} \frac{d \boldsymbol{p}_{2}^{\prime}}{E_{\boldsymbol{p}_{2}^{\prime}}} \frac{d \boldsymbol{p}_{1}}{E_{\boldsymbol{p}_{1}}} \frac{d \boldsymbol{p}_{2}}{E_{\boldsymbol{p}_{2}}} \times$

$\times V_{\gamma}\left(p_{1}^{\prime}, p_{2}^{\prime} ; p_{1}, p_{2}\right) b_{c}^{\dagger}\left(p_{1}^{\prime}\right) b_{c}^{\dagger}\left(p_{2}^{\prime}\right) b_{c}\left(p_{1}\right) b_{c}\left(p_{2}\right)$,

with the $c$-number matrix

$V_{\gamma}\left(p_{1}^{\prime}, p_{2}^{\prime} ; p_{1}, p_{2}\right)=\frac{e^{2} m^{2}}{(2 \pi)^{3}} \delta\left(\boldsymbol{p}_{2}^{\prime}+\boldsymbol{p}_{1}^{\prime}-\boldsymbol{p}_{2}-\boldsymbol{p}_{1}\right) \times$

$\times v\left(p_{1}^{\prime}, p_{2}^{\prime} ; p_{1}, p_{2}\right)$,

$v\left(p_{1}^{\prime}, p_{2}^{\prime} ; p_{1}, p_{2}\right)=-\frac{1}{2} \frac{\bar{u}\left(p_{1}^{\prime}\right) \gamma^{\mu} u\left(p_{1}\right) \bar{u}\left(p_{2}^{\prime}\right) \gamma^{\nu} u\left(p_{2}\right)}{\left(p_{1}-p_{1}^{\prime}\right)^{2}-\lambda^{2}} \times$

$\times P_{\mu \nu}\left(p_{1}^{\prime}-p_{1}\right)$.

When deriving these formulae, we have used the condition (8), the Dirac equation $(\not p-m) u(p)=0$, and the representation

$V_{\text {Coul } e^{-} e^{-} \rightarrow e^{-} e^{-}}=\int \frac{d \boldsymbol{p}_{1}^{\prime}}{E_{\boldsymbol{p}_{1}^{\prime}}} \frac{d \boldsymbol{p}_{2}^{\prime}}{E_{\boldsymbol{p}_{2}^{\prime}}} \frac{d \boldsymbol{p}_{1}}{E_{\boldsymbol{p}_{1}}} \frac{d \boldsymbol{p}_{2}}{E_{\boldsymbol{p}_{2}}} \times$

$\times V_{\text {Coul }}\left(p_{1}^{\prime}, p_{2}^{\prime} ; p_{1}, p_{2}\right) b_{c}^{\dagger}\left(p_{1}^{\prime}\right) b_{c}^{\dagger}\left(p_{2}^{\prime}\right) b_{c}\left(p_{1}\right) b_{c}\left(p_{2}\right)$, 
$V_{\text {Coul }}\left(p_{1}^{\prime}, p_{2}^{\prime} ; p_{1}, p_{2}\right)=\frac{e^{2} m^{2}}{(2 \pi)^{3}} \delta\left(\boldsymbol{p}_{2}^{\prime}+\boldsymbol{p}_{1}^{\prime}-\boldsymbol{p}_{2}-\boldsymbol{p}_{1}\right) \times$

$\times v_{\text {Coul }}\left(p_{1}^{\prime}, p_{2}^{\prime} ; p_{1}, p_{2}\right)$,

$v_{\text {Coul }}\left(p_{1}^{\prime}, p_{2}^{\prime} ; p_{1}, p_{2}\right)=-\frac{1}{2} \frac{\bar{u}\left(p_{1}^{\prime}\right) \gamma^{0} u\left(p_{1}\right) \bar{u}\left(p_{2}^{\prime}\right) \gamma^{0} u\left(p_{2}\right)}{\left(\boldsymbol{p}_{1}-\boldsymbol{p}_{1}^{\prime}\right)^{2}+\lambda^{2}}$.

Moreover, we do not hurry to put $\lambda=0$. Besides, keeping in mind the problem of removing the infrared divergences, it is convenient sometimes to handle an infinitesimally small photon mass.

Thus, within our approach, the two-clothedelectron interaction given by Eq. (13) acquires a covariant form due to the cancellation of the non-covariant primary Coulomb interaction contribution to the QED Hamiltonian. In our opinion, it is the time to quote from [14] on p. 355, viz., "... the apparent violation of Lorentz invariance in the instantaneous Coulomb interaction is cancelled by another apparent violation of Lorentz invariance, ..." that arises, since the photon fields $A_{D}^{\mu}(x)$ do not transform as fourvectors, "and therefore have a non-covariant propagator". An important point is that, in CPR, unlike [14], such a cancellation (see also our results [6] in mesodynamics) takes place directly in the Hamiltonian, when starting with the CG. Such a distinct feature of the UCT method makes it useful in covariant calculations of the $S$-matrix either by solving the two-particle Lippmann-Schwinger equation (LSE) for the corresponding $T$-matrix or using the perturbation theory (not obligatorily addressing the Dyson-Feynman expansion). In this context, we would like to note an akin approach developed in [16] to problems of the relativistic QFT that deserves, in our opinion, a very undiverted attention.

Of course, doing so, one can find not only the $S$-matrix, but the eigenstates of the operator $K=$ $=K_{F}+K_{I}$ in the Fock subspace $R_{F}^{[2]}$ spanned onto the clothed-two-particle $K_{F}$ eigenvectors. In this connection, one has to deal with

$$
\begin{aligned}
& K_{F}=\int \frac{d \boldsymbol{k}}{\omega_{\boldsymbol{k}}} \omega_{\boldsymbol{k}} c_{c}^{\dagger}(k) c_{c}(k)+ \\
& +\int \frac{d \boldsymbol{p}}{E_{\boldsymbol{p}}} E_{\boldsymbol{p}}\left[b_{c}^{\dagger}(p) b_{c}(p)+d_{c}^{\dagger}(p) d_{c}(p)\right] .
\end{aligned}
$$

\section{Explicit Expressions for the Interaction Operators}

In our previous works $[12,13]$, we have already presented the expressions for $K_{e^{-} e^{+} \rightarrow e^{-} e^{+}}$and
$K_{e^{-} e^{+} \rightarrow \gamma \gamma}$ when deriving the first correction to the positronium ground state energy and its decay to two photons. Now, we will show all the new $e^{2}$-order interactions between the clothed electrons, positrons, and photons in terms of the clothed destruction (creation) operators $b_{c}, d_{c}, c_{c}\left(b_{c}^{\dagger}, d_{c}^{\dagger}, c_{c}^{\dagger}\right)$ compared to the expressions obtained within the Feynman approach in terms of the bare destruction (creation) operators $b$, $d, c\left(b^{\dagger}, d^{\dagger}, c^{\dagger}\right)$.

\subsection{The interaction operator for $e^{-} e^{-}$-scattering}

To get the operator $K_{e^{-} e^{-} \rightarrow e^{-} e^{-}}$, one needs to separate out the $b_{c}^{\dagger} b_{c}^{\dagger} b_{c} b_{c}$-type terms from the Hamiltonian $K\left(\alpha_{c}\right)$. Uniting Eqs. (16 and 17), we arrive at

$$
\begin{aligned}
& K_{e^{-} e^{-} \rightarrow e^{-} e^{-}}=\int \frac{d \boldsymbol{p}_{1}^{\prime}}{E_{\boldsymbol{p}_{1}^{\prime}}} \frac{d \boldsymbol{p}_{2}^{\prime}}{E_{\boldsymbol{p}_{2}^{\prime}}} \frac{d \boldsymbol{p}_{1}}{E_{\boldsymbol{p}_{1}}} \frac{d \boldsymbol{p}_{2}}{E_{\boldsymbol{p}_{2}}} \times \\
& \times V_{e^{-} e^{-}}\left(p_{1}^{\prime}, p_{2}^{\prime} ; p_{1}, p_{2}\right) b_{c}^{\dagger}\left(p_{1}^{\prime}\right) b_{c}^{\dagger}\left(p_{2}^{\prime}\right) b_{c}\left(p_{1}\right) b_{c}\left(p_{2}\right), \\
& V_{e^{-} e^{-}}\left(p_{1}^{\prime}, p_{2}^{\prime} ; p_{1}, p_{2}\right)=\frac{e^{2} m^{2}}{(2 \pi)^{3}} \delta\left(\boldsymbol{p}_{2}^{\prime}+\boldsymbol{p}_{1}^{\prime}-\boldsymbol{p}_{2}-\boldsymbol{p}_{1}\right) \times \\
& \times\left[v_{e^{-} e^{-}}^{\text {Feynan-like }}+v_{e^{-} e^{-}}^{\text {off-ergy-shell }}\right], \\
& v_{e^{-} e^{-}}^{\text {Feynman-like }}=\frac{1}{2} \frac{\bar{u}\left(p_{1}^{\prime}\right) \gamma^{\mu} u\left(p_{1}\right) \bar{u}\left(p_{2}^{\prime}\right) \gamma_{\mu} u\left(p_{2}\right)}{\left(p_{1}^{\prime}-p_{1}\right)^{2}-\lambda^{2}}, \\
& v_{e^{-} e^{-}}^{\text {off-energy-shell }}=-\frac{1}{2} \frac{\left(p_{1}^{\prime}+p_{2}^{\prime}-p_{1}-p_{2}\right)\left(p_{1}^{\prime}-p_{1}\right)}{\left(\boldsymbol{p}_{1}^{\prime}-\boldsymbol{p}_{1}\right)^{2}+\lambda^{2}} \times \\
& \times \frac{\bar{u}\left(p_{1}^{\prime}\right) \gamma^{0} u\left(p_{1}\right) \bar{u}\left(p_{2}^{\prime}\right) \gamma^{0} u\left(p_{2}\right)}{\left(p_{1}^{\prime}-p_{1}\right)^{2}-\lambda^{2}} .
\end{aligned}
$$

Here, all momenta are defined on the mass-shell: $p^{2}=E_{\boldsymbol{p}}^{2}-\boldsymbol{p}^{2}=m^{2}$. The Feynman-type propagator $\left(\left(p_{1}^{\prime}-p_{1}\right)^{2}-\lambda^{2}\right)^{-1}$ in (21) stems from adding the two noncovariant ("nonrelativistic") propagators

$$
\begin{aligned}
& \frac{1}{2 \omega_{\boldsymbol{p}_{1}^{\prime}}-\boldsymbol{p}_{1}}\left\{\frac{1}{E_{\boldsymbol{p}_{1}}-E_{\boldsymbol{p}_{1}^{\prime}}-\omega_{\boldsymbol{p}_{1}^{\prime}-\boldsymbol{p}_{1}}}+\right. \\
& \left.+\frac{1}{E_{\boldsymbol{p}_{1}^{\prime}}-E_{\boldsymbol{p}_{1}}-\omega_{\boldsymbol{p}_{1}^{\prime}-\boldsymbol{p}_{1}}}\right\},
\end{aligned}
$$

with $\omega_{\boldsymbol{p}_{1}^{\prime}-\boldsymbol{p}_{1}}=\sqrt{\left(\boldsymbol{p}_{1}^{\prime}-\boldsymbol{p}_{1}\right)^{2}+\lambda^{2}}$. Such a feature of the UCT method allows us to use the graphic language of the old-fashioned perturbation theory (OFPT) (see, e.g., Chapter 13 in [17]), when addressing the graphs in Fig. 1. As noted in [5], the graphs in Fig. 1 are topologically equivalent to the time-ordered Feynman diagram in Fig. 2. However, 

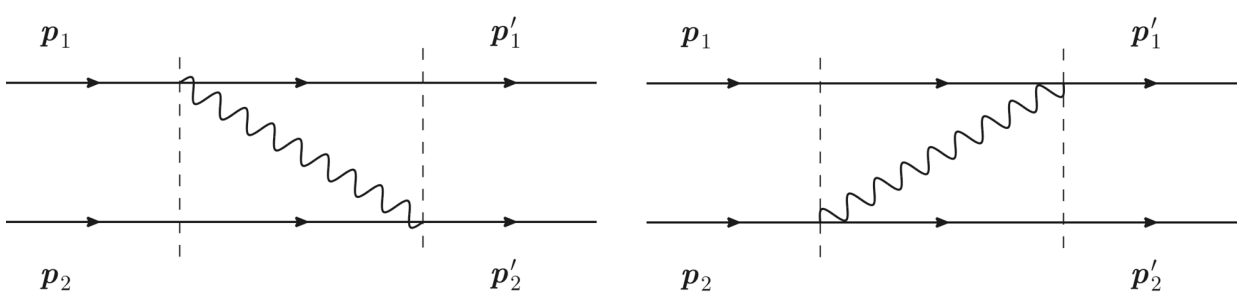

Fig. 1. The typical OFPT diagrams with the intermediate photon (wavy line) on its mass shell

in the Schrödinger picture employed here, where all events are related to the same instant $t=0$, such an analogy seems to be misleading. In the OFPT, the particle three-momenta are related to the lines in Fig. 1 via the following prescription: when moving from the left to the right, a sum of "outgoing" momenta is equal to a sum of "ingoing" ones. Here, the term "ingoing" (outgoing) is referred to the corresponding line or lines lying on the left (right) from the dotted vertical (a "phantom" line) that passes through a given vertex. In fact, the line directions in, fore example, Fig. 3 are given with the sole scope to discriminate between the fermion and antifermion states.

In this way, the denominator $\left(E_{\boldsymbol{p}_{1}}-E_{\boldsymbol{p}_{1}^{\prime}}-\omega_{\boldsymbol{p}_{1}^{\prime}-\boldsymbol{p}_{1}}\right)^{-1}$ may be related to the noncovariant propagator

$D^{-1}(E)_{E=E_{\boldsymbol{p}_{1}}+E_{\boldsymbol{p}_{2}}} \equiv$

$\left.\equiv\left(E-E_{\boldsymbol{p}_{1}^{\prime}}-E_{\boldsymbol{p}_{2}}-\omega_{\boldsymbol{p}_{1}^{\prime}-\boldsymbol{p}_{1}}\right)\right|_{E=E_{\boldsymbol{p}_{1}}+E_{\boldsymbol{p}_{2}}}=$

$=E_{\boldsymbol{p}_{1}}-E_{\boldsymbol{p}_{1}^{\prime}}-\omega_{\boldsymbol{p}_{1}^{\prime}-\boldsymbol{p}_{1}}$,

which is associated with the right diagram in Fig. 1, where the three-momentum is conserved at the each vertex. Such an interpretation has much in common with the theory of meson-nucleon interactions (see Sec. 3.2 in [5]).

Furthermore, according to $[3,5]$, the properly symmetrized interaction is given by the matrix element (quasipotential)

$$
\begin{aligned}
& \bar{V}_{e^{-} e^{-}}\left(p_{1}^{\prime}, p_{2}^{\prime} ; p_{1}, p_{2}\right)= \\
& =\left\langle b_{c}^{\dagger}\left(p_{1}^{\prime}\right) b_{c}^{\dagger}\left(p_{2}^{\prime}\right) \Omega\left|K_{e^{-} e^{-} \rightarrow e^{-} e^{-}}\right| b_{c}^{\dagger}\left(p_{1}\right) b_{c}^{\dagger}\left(p_{2}\right) \Omega\right\rangle= \\
& =-V_{e^{-} e^{-}}\left(p_{1}^{\prime}, p_{2}^{\prime} ; p_{1}, p_{2}\right)-V_{e^{-} e^{-}}\left(p_{2}^{\prime}, p_{1}^{\prime} ; p_{2}, p_{1}\right)+ \\
& +V_{e^{-} e^{-}}\left(p_{2}^{\prime}, p_{1}^{\prime} ; p_{1}, p_{2}\right)+V_{e^{-} e^{-}}\left(p_{1}^{\prime}, p_{2}^{\prime} ; p_{2}, p_{1}\right)= \\
& =\frac{e^{2} m^{2}}{(2 \pi)^{3}} \delta\left(\boldsymbol{p}_{2}^{\prime}+\boldsymbol{p}_{1}^{\prime}-\boldsymbol{p}_{2}-\boldsymbol{p}_{1}\right) \times \\
& \times\left[\bar{v}_{e^{-} e^{-}}^{\text {Feynman-like }}+\bar{v}_{e^{-} e^{-}}^{\text {off-energy-shell }}\right],
\end{aligned}
$$

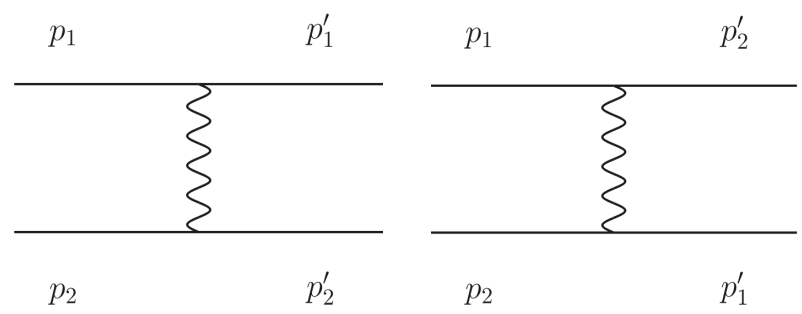

Fig. 2. On-energy-shell contributions to the electron-electron interaction (the $e^{2}$-order Feynman diagrams for the electronelectron scattering)

$\bar{v}_{e^{-} e^{-}}^{\text {Feynman-like }}=-\bar{u}\left(p_{1}^{\prime}\right) \gamma^{\mu} u\left(p_{1}\right) \times$

$\times \frac{1}{2}\left\{\frac{1}{\left(p_{1}^{\prime}-p_{1}\right)^{2}-\lambda^{2}}+\frac{1}{\left(p_{2}^{\prime}-p_{2}\right)^{2}-\lambda^{2}}\right\} \times$

$\times \bar{u}\left(p_{2}^{\prime}\right) \gamma_{\mu} u\left(p_{2}\right)-(1 \leftrightarrow 2)$,

$\bar{v}_{e^{-} e^{-}}^{\text {off-energy-shell }}=\frac{\left(p_{1}^{\prime}+p_{2}^{\prime}-p_{1}-p_{2}\right)}{\left(\boldsymbol{p}_{1}^{\prime}-\boldsymbol{p}_{1}\right)^{2}+\lambda^{2}} \bar{u}\left(p_{1}^{\prime}\right) \gamma^{0} u\left(p_{1}\right) \times$

$\times \frac{1}{2}\left\{\frac{\left(p_{1}^{\prime}-p_{1}\right)}{\left(p_{1}^{\prime}-p_{1}\right)^{2}-\lambda^{2}}+\frac{\left(p_{2}^{\prime}-p_{2}\right)}{\left(p_{2}^{\prime}-p_{2}\right)^{2}-\lambda^{2}}\right\} \times$

$\times \bar{u}\left(p_{2}^{\prime}\right) \gamma^{0} u\left(p_{2}\right)-(1 \leftrightarrow 2)$.

The r.h.s. of these equations consists of the "direct" term written explicitly and the "exchange" one $(1 \leftrightarrow 2)$ with the prescription that the variables with labels 1 and 2 should be mutually interchanged.

Unlike the interactions built up in mesodynamics, a distinctive feature is the appearance of the noncovariant contribution (27) due to the interplay between the Coulomb interaction (4) and the noncovariant term from $\frac{1}{2}\left[R, V^{(1)}\right]_{b_{c}^{\dagger} b_{c}^{\dagger} b_{c} b_{c}}$. One should note that on the energy shell for the electron-electron scattering, i.e., under the condition

$E_{\boldsymbol{p}_{1}^{\prime}}+E_{\boldsymbol{p}_{2}^{\prime}}=E_{\boldsymbol{p}_{1}}+E_{\boldsymbol{p}_{2}}$

this contribution becomes to be zero. The important point is that such a cancellation in CPR takes 
place directly in the Hamiltonian. In this context, one should stress that, on the energy shell, only the Feynman-like contribution (26) remains, and the expression in the curly brackets

$$
\frac{1}{2}\left\{\frac{1}{\left(p_{1}^{\prime}-p_{1}\right)^{2}-\lambda^{2}}+\frac{1}{\left(p_{2}^{\prime}-p_{2}\right)^{2}-\lambda^{2}}\right\}
$$

is converted into the genuine Feynman propagator which occurs, when the $S$-operator in the $e^{2}$-order

$S_{e^{-} e^{-} \rightarrow e^{-} e^{-}}^{(2)}=\int \frac{d \boldsymbol{p}_{1}^{\prime}}{E_{\boldsymbol{p}_{1}^{\prime}}} \frac{d \boldsymbol{p}_{2}^{\prime}}{E_{\boldsymbol{p}_{2}^{\prime}}} \frac{d \boldsymbol{p}_{1}}{E_{\boldsymbol{p}_{1}}} \frac{d \boldsymbol{p}_{2}}{E_{\boldsymbol{p}_{2}}} \times$

$\times V_{e^{-} e^{-}}^{\text {Feynman }}\left(p_{1}^{\prime}, p_{2}^{\prime} ; p_{1}, p_{2}\right) b^{\dagger}\left(p_{1}^{\prime}\right) b^{\dagger}\left(p_{2}^{\prime}\right) b\left(p_{1}\right) b\left(p_{2}\right)$,

$V_{e^{-} e^{-}}^{\text {Feynman }}\left(p_{1}^{\prime}, p_{2}^{\prime} ; p_{1}, p_{2}\right)=-i \frac{e_{0}^{2} m_{0}^{2}}{(2 \pi)^{2}} \times$

$\times \delta\left(p_{2}^{\prime}+p_{1}^{\prime}-p_{2}-p_{1}\right) v_{e^{-} e^{-}}^{\text {Feynman-like }}\left(m \rightarrow m_{0}\right)$,

is sandwiched between the bare states $\left\langle b^{\dagger}\left(p_{1}^{\prime}\right) b^{\dagger}\left(p_{2}^{\prime}\right) \times\right.$ $\left.\times \Omega_{0}\left|S^{(2)}\right| b^{\dagger}\left(p_{1}\right) b^{\dagger}\left(p_{2}\right) \Omega_{0}\right\rangle$. The corresponding diagrams are displayed in Fig. 2.

It is important to emphasize that interaction (25) is determined not only on the energy shell, but also beyond it, because it does not contain the factor $\delta\left(E_{\boldsymbol{p}_{1}}+E_{\boldsymbol{p}_{2}}-E_{\boldsymbol{p}_{1}^{\prime}}-E_{\boldsymbol{p}_{2}^{\prime}}\right)$. This is a curious feature of the UCT method. The interaction obtained is nonlocal, since the vertex factors and propagators in Eqs. (26) and (27) are dependent not only on the relative three-momenta involved, but also on their total three-momentum. The interactions in [5] have the same property.

\subsection{The interaction operator for $e^{-} e^{+}$-scattering}

Next, it is the operator of $e^{-} e^{+}$scattering. Now, we are interested in the $b_{c}^{\dagger} d_{c}^{\dagger} b_{c} d_{c}$-type terms

$$
\begin{aligned}
& K_{e^{-} e^{+} \rightarrow e^{-} e^{+}}=\int \frac{d \boldsymbol{p}_{1}^{\prime}}{E_{\boldsymbol{p}_{1}^{\prime}}} \frac{d \boldsymbol{p}_{2}^{\prime}}{E_{\boldsymbol{p}_{2}^{\prime}}} \frac{d \boldsymbol{p}_{1}}{E_{\boldsymbol{p}_{1}}} \frac{d \boldsymbol{p}_{2}}{E_{\boldsymbol{p}_{2}}} \times \\
& \times V_{e^{-} e^{+}}\left(p_{1}^{\prime}, p_{2}^{\prime} ; p_{1}, p_{2}\right) b_{c}^{\dagger}\left(p_{1}^{\prime}\right) d_{c}^{\dagger}\left(p_{2}^{\prime}\right) b_{c}\left(p_{1}\right) d_{c}\left(p_{2}\right), \\
& V_{e^{-} e^{+}}\left(p_{1}^{\prime}, p_{2}^{\prime} ; p_{1}, p_{2}\right)=\frac{e^{2} m^{2}}{(2 \pi)^{3}} \delta\left(\boldsymbol{p}_{2}^{\prime}+\boldsymbol{p}_{1}^{\prime}-\boldsymbol{p}_{2}-\boldsymbol{p}_{1}\right) \times \\
& \times\left[v_{S}\left(p_{1}^{\prime}, p_{2}^{\prime} ; p_{1}, p_{2}\right)+v_{A}\left(p_{1}^{\prime}, p_{2}^{\prime} ; p_{1}, p_{2}\right)\right] \\
& v_{S / A}=v_{S / A}^{\text {Feynman-like }}+v_{S / A}^{\text {off-energy-shell }}
\end{aligned}
$$

\section{8}

$$
\begin{aligned}
& v_{S}^{\text {Feynman-like }}=-\bar{u}\left(p_{1}^{\prime}\right) \gamma^{\mu} u\left(p_{1}\right) \frac{1}{2}\left\{\frac{1}{\left(p_{1}^{\prime}-p_{1}\right)^{2}-\lambda^{2}}+\right. \\
& \left.+\frac{1}{\left(p_{2}^{\prime}-p_{2}\right)^{2}-\lambda^{2}}\right\} \bar{v}\left(p_{2}\right) \gamma_{\mu} v\left(p_{2}^{\prime}\right), \\
& v_{S}^{\text {off-energy-shell }}=\frac{\left(p_{1}^{\prime}+p_{2}^{\prime}-p_{1}-p_{2}\right)}{\left(\boldsymbol{p}_{1}^{\prime}-\boldsymbol{p}_{1}\right)^{2}+\lambda^{2}} \bar{u}\left(p_{1}^{\prime}\right) \gamma^{0} u\left(p_{1}\right) \times \\
& \times \frac{1}{2}\left\{\frac{\left(p_{1}^{\prime}-p_{1}\right)}{\left(p_{1}^{\prime}-p_{1}\right)^{2}-\lambda^{2}}+\frac{\left(p_{2}^{\prime}-p_{2}\right)}{\left(p_{2}^{\prime}-p_{2}\right)^{2}-\lambda^{2}}\right\} \times \\
& \times \bar{v}\left(p_{2}\right) \gamma^{0} v\left(p_{2}^{\prime}\right) \\
& v_{A}^{\text {Feynman-like }}=\bar{u}\left(p_{1}^{\prime}\right) \gamma^{\mu} v\left(p_{2}^{\prime}\right) \frac{1}{2}\left\{\frac{1}{\left(p_{1}+p_{2}\right)^{2}-\lambda^{2}}+\right. \\
& \left.+\frac{1}{\left(p_{1}^{\prime}+p_{2}^{\prime}\right)^{2}-\lambda^{2}}\right\} \bar{v}\left(p_{2}\right) \gamma_{\mu} u\left(p_{1}\right), \\
& v_{A}^{\text {off-energy-shell }}=-\frac{\left(p_{1}^{\prime}+p_{2}^{\prime}-p_{1}-p_{2}\right)}{\left(\boldsymbol{p}_{1}^{\prime}+\boldsymbol{p}_{2}^{\prime}\right)^{2}+\lambda^{2}} \bar{u}\left(p_{1}^{\prime}\right) \gamma^{0} v\left(p_{2}^{\prime}\right) \times \\
& \times \frac{1}{2}\left\{\frac{\left(p_{1}^{\prime}+p_{2}^{\prime}\right)}{\left(p_{1}^{\prime}+p_{2}^{\prime}\right)^{2}-\lambda^{2}}-\frac{\left(p_{1}+p_{2}\right)}{\left(p_{1}+p_{2}\right)^{2}-\lambda^{2}}\right\} \times \\
& \times \bar{v}\left(p_{2}\right) \gamma^{0} u\left(p_{1}\right) \text {. }
\end{aligned}
$$

We have introduced the decomposition into the socalled scattering and annihilation contributions $v_{S}$ and $v_{A}$. Each of them has structure (34). Again, only the Feynman-like part survives on the energy-shell, i.e., under the condition $p_{1}^{\prime 0}+p_{2}^{\prime 0}=p_{1}^{0}+p_{2}^{0}$.

The corresponding $S$-operator is

$$
\begin{aligned}
& S_{e^{-} e^{+} \rightarrow e^{-} e^{+}}=\int \frac{d \boldsymbol{p}_{1}^{\prime}}{E_{\boldsymbol{p}_{1}^{\prime}}} \frac{d \boldsymbol{p}_{2}^{\prime}}{E_{\boldsymbol{p}_{2}^{\prime}}} \frac{d \boldsymbol{p}_{1}}{E_{\boldsymbol{p}_{1}}} \frac{d \boldsymbol{p}_{2}}{E_{\boldsymbol{p}_{2}}} \times \\
& \times V_{e^{-} e^{+}}^{\mathrm{Feynman}}\left(p_{1}^{\prime}, p_{2}^{\prime} ; p_{1}, p_{2}\right) b^{\dagger}\left(p_{1}^{\prime}\right) d^{\dagger}\left(p_{2}^{\prime}\right) b\left(p_{1}\right) d\left(p_{2}\right),
\end{aligned}
$$

$$
\begin{aligned}
& V_{e^{-} e^{+}}^{\text {Feynman }}\left(p_{1}^{\prime}, p_{2}^{\prime} ; p_{1}, p_{2}\right)=-i \frac{e_{0}^{2} m_{0}^{2}}{(2 \pi)^{2}} \delta\left(p_{2}^{\prime}+p_{1}^{\prime}-p_{2}-p_{1}\right) \times \\
& \times\left[v_{S}^{\text {Feynman }}\left(p_{1}^{\prime}, p_{2}^{\prime} ; p_{1}, p_{2}\right)+v_{A}^{\text {Feynman }}\left(p_{1}^{\prime}, p_{2}^{\prime} ; p_{1}, p_{2}\right)\right], \\
& v_{S}^{\text {Feynman }}=-\bar{u}\left(p_{1}^{\prime}\right) \gamma^{\mu} u\left(p_{1}\right) \frac{1}{\left(p_{1}^{\prime}-p_{1}\right)^{2}} \bar{v}\left(p_{2}\right) \gamma_{\mu} v\left(p_{2}^{\prime}\right), \\
& v_{A}^{\text {Feynman }}=\bar{u}\left(p_{1}^{\prime}\right) \gamma^{\mu} v\left(p_{2}^{\prime}\right) \frac{1}{\left(p_{1}+p_{2}\right)^{2}} \bar{v}\left(p_{2}\right) \gamma_{\mu} u\left(p_{1}\right),
\end{aligned}
$$

with the bare electron mass $m_{0}$ and charge $e_{0}$.

The scattering term $v_{S}$ can be obtained from the direct parts of Eqs. $(26,27)$

$\bar{u}\left(p_{2}^{\prime}\right) \rightarrow \bar{v}\left(p_{2}\right), \quad u\left(p_{2}\right) \rightarrow v\left(p_{2}^{\prime}\right)$,

ISSN 2071-0194. Ukr. J. Phys. 2021. Vol. 66, No. 10 

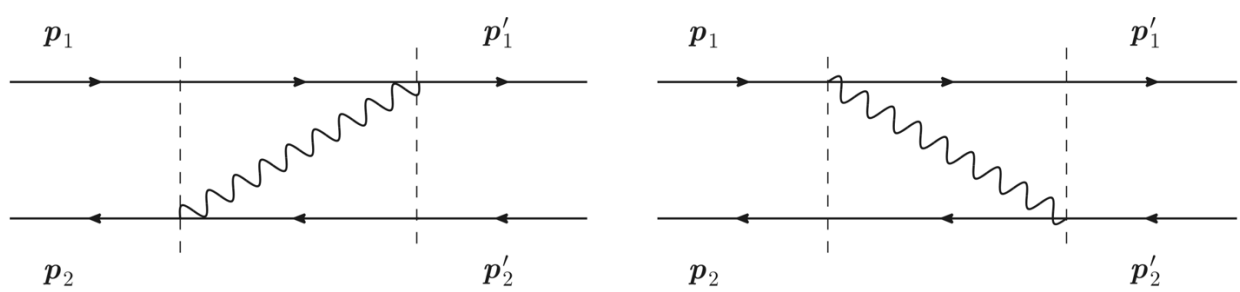

Fig. 3. OFPT graphs for the scattering contribution $\left(v_{S}\right)$ to the electron-positron scattering process
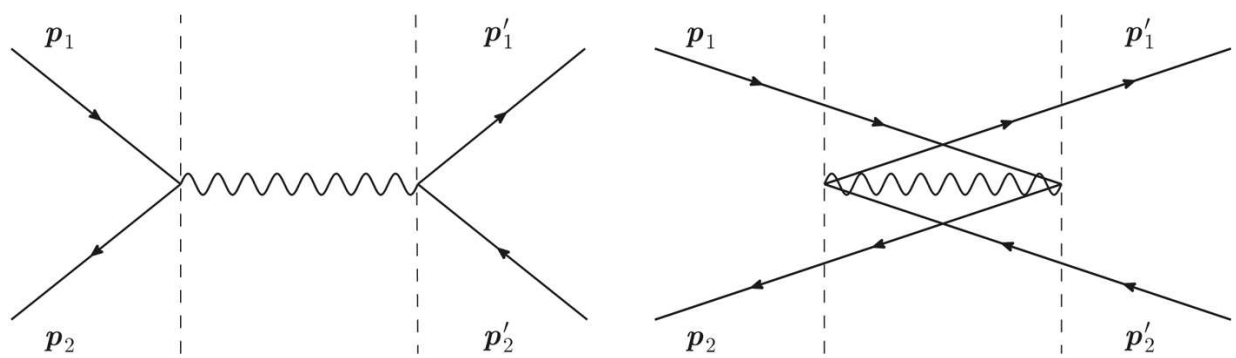

Fig. 4. OFPT graphs for the annihilation contribution $\left(v_{A}\right)$ to the electron-positron scattering process

and the corresponding OFPT graphs are obtained from the Fig. 1 by replacing the electron line with the positron one that is illustrated in Fig. 3. Henceforth, the line directions in graphs are given with the sole scope to discriminate between the electron and positron states.

In the annihilation term, the Feynman-like propagators arise from the noncovariant propagators in the following way (see details in [18])

$$
\begin{aligned}
& \frac{1}{\omega_{\boldsymbol{p}_{1}^{\prime}+\boldsymbol{p}_{2}^{\prime}}-E_{\boldsymbol{p}_{1}^{\prime}}-E_{\boldsymbol{p}_{2}^{\prime}}}+\frac{1}{\omega_{\boldsymbol{p}_{1}^{\prime}+\boldsymbol{p}_{2}^{\prime}}+E_{\boldsymbol{p}_{1}^{\prime}}+E_{\boldsymbol{p}_{2}^{\prime}}} \rightarrow \\
& \rightarrow-\frac{1}{\left(p_{1}^{\prime}+p_{2}^{\prime}\right)^{2}-\lambda^{2}}, \\
& \frac{1}{\omega_{\boldsymbol{p}_{1}+\boldsymbol{p}_{2}}-E_{\boldsymbol{p}_{1}}-E_{\boldsymbol{p}_{2}}}+\frac{1}{\omega_{\boldsymbol{p}_{1}+\boldsymbol{p}_{2}}+E_{\boldsymbol{p}_{1}}+E_{\boldsymbol{p}_{2}}} \rightarrow \\
& \rightarrow-\frac{1}{\left(p_{1}+p_{2}\right)^{2}-\lambda^{2}} .
\end{aligned}
$$

The two noncovariant denominators

$\left.D_{1}^{-1}(E)_{E=E_{\boldsymbol{p}_{1}}+E_{\boldsymbol{p}_{2}}} \equiv\left(E-\omega_{\boldsymbol{p}_{1}+\boldsymbol{p}_{2}}\right)\right|_{E=E_{\boldsymbol{p}_{1}}+E_{\boldsymbol{p}_{2}}}=$

$=-\left(\omega_{\boldsymbol{p}_{1}+\boldsymbol{p}_{2}}-E_{\boldsymbol{p}_{1}}-E_{\boldsymbol{p}_{2}}\right)$,

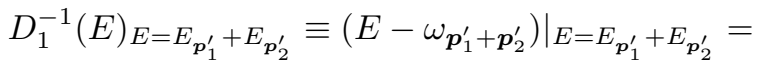

$=-\left(\omega_{\boldsymbol{p}_{1}^{\prime}+\boldsymbol{p}_{2}^{\prime}}-E_{\boldsymbol{p}_{1}^{\prime}}-E_{\boldsymbol{p}_{2}^{\prime}}\right)$

ISSN 2071-0194. Ukr. J. Phys. 2021. Vol. 66, No. 10 can be associated with the left graph in Fig. 4 with one internal line putting between the dotted ("phantom") lines. In its turn, the right graph with five internal lines relates to the denominators

$D_{2}^{-1}(E)_{E=E_{\boldsymbol{p}_{1}}+E_{\boldsymbol{p}_{2}}} \equiv$

$\left.\equiv\left(E-E_{\boldsymbol{p}_{1}^{\prime}}-E_{\boldsymbol{p}_{2}^{\prime}}-E_{\boldsymbol{p}_{1}}-E_{\boldsymbol{p}_{2}}-\omega_{\boldsymbol{p}_{1}^{\prime}+\boldsymbol{p}_{2}^{\prime}}\right)\right|_{E=E_{\boldsymbol{p}_{1}}+E_{\boldsymbol{p}_{2}}}=$

$=-\left(\omega_{\boldsymbol{p}_{1}^{\prime}+\boldsymbol{p}_{2}^{\prime}}-E_{\boldsymbol{p}_{1}^{\prime}}-E_{\boldsymbol{p}_{2}^{\prime}}\right)$,

$D_{2}^{-1}(E)_{E=E_{\boldsymbol{p}_{1}^{\prime}}+E_{\boldsymbol{p}_{2}^{\prime}}} \equiv$

$\left.\equiv\left(E-E_{\boldsymbol{p}_{1}^{\prime}}-E_{\boldsymbol{p}_{2}^{\prime}}-E_{\boldsymbol{p}_{1}}-E_{\boldsymbol{p}_{2}}-\omega_{\boldsymbol{p}_{1}+\boldsymbol{p}_{2}}\right)\right|_{E=E_{\boldsymbol{p}_{1}^{\prime}}+E_{\boldsymbol{p}_{2}^{\prime}}}=$

$=\left(\omega_{\boldsymbol{p}_{1}+\boldsymbol{p}_{2}}-E_{\boldsymbol{p}_{1}}-E_{\boldsymbol{p}_{2}}\right)$.

By analogy with the interaction between clothed electrons, we introduce the matrix element

$\bar{V}_{e^{-} e^{+}}\left(p_{1}^{\prime}, p_{2}^{\prime} ; p_{1}, p_{2}\right)=$

$=\left\langle b_{c}^{\dagger}\left(p_{1}^{\prime}\right) d_{c}^{\dagger}\left(p_{2}^{\prime}\right) \Omega\left|K_{e^{-} e^{+} \rightarrow e^{-} e^{+}}\right| b_{c}^{\dagger}\left(p_{1}\right) d_{c}^{\dagger}\left(p_{2}\right) \Omega\right\rangle=$

$=-V_{e^{-} e^{+}}\left(p_{1}^{\prime}, p_{2}^{\prime} ; p_{1}, p_{2}\right)$,

which is equal to Eq. (33) with the opposite sign. Like the case of the electron-electron interaction, the denominators in the curly brackets in the Feynmanlike contributions are converted into the genuine 

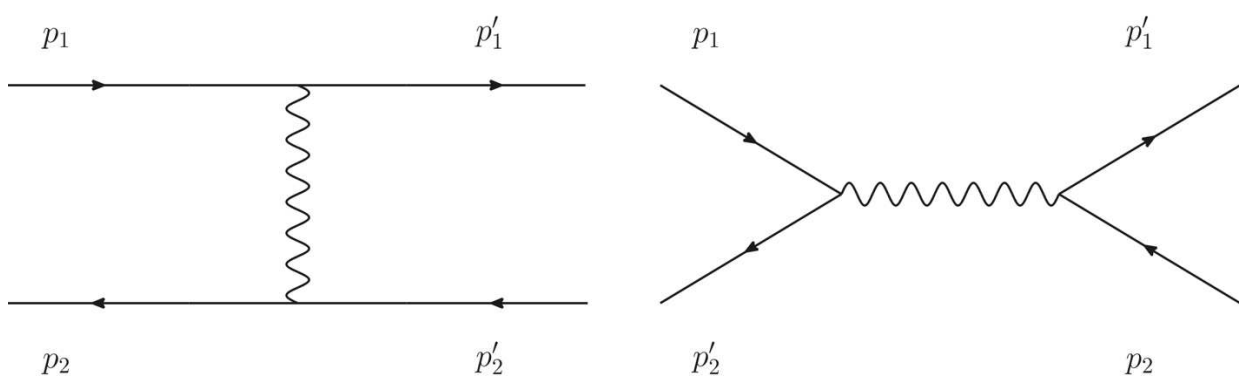

Fig. 5. Feynman diagrams for the on-shell contribution to the electron-positron interaction

Feynman propagators

$$
\begin{aligned}
& \frac{1}{2}\left\{\frac{1}{\left(p_{1}^{\prime}-p_{1}\right)^{2}-\lambda^{2}}+\frac{1}{\left(p_{2}^{\prime}-p_{2}\right)^{2}-\lambda^{2}}\right\} \rightarrow \\
& \rightarrow \frac{1}{\left(p_{1}^{\prime}-p_{1}\right)^{2}-\lambda^{2}}=\frac{1}{\left(p_{2}^{\prime}-p_{2}\right)^{2}-\lambda^{2}}, \\
& \frac{1}{2}\left\{\frac{1}{\left(p_{1}+p_{2}\right)^{2}-\lambda^{2}}+\frac{1}{\left(p_{1}^{\prime}+p_{2}^{\prime}\right)^{2}-\lambda^{2}}\right\} \rightarrow \\
& \rightarrow \frac{1}{\left(p_{1}^{\prime}+p_{2}^{\prime}\right)^{2}-\lambda^{2}}=\frac{1}{\left(p_{1}+p_{2}\right)^{2}-\lambda^{2}} .
\end{aligned}
$$

The corresponding Feynman graphs are displayed in Fig. 5.

\subsection{The interaction operator for $e^{-} e^{+}$-pair annihilation}

The interaction operator corresponding to the annihilation of the clothed electron and positron to two photons is

$$
\begin{aligned}
& K_{e^{-} e^{+} \rightarrow \gamma \gamma}=\int \frac{d \boldsymbol{k}_{1}}{\omega_{\boldsymbol{k}_{1}}} \frac{d \boldsymbol{k}_{2}}{\omega_{\boldsymbol{k}_{2}}} \frac{d \boldsymbol{p}_{1}}{E_{\boldsymbol{p}_{1}}} \frac{d \boldsymbol{p}_{2}}{E_{\boldsymbol{p}_{2}}} \times \\
& \times V_{e^{-} e^{+} \rightarrow \gamma \gamma}\left(k_{2}, k_{1} ; p_{2}, p_{1}\right) c_{c}^{\dagger}\left(k_{2}\right) c_{c}^{\dagger}\left(k_{1}\right) b_{c}\left(p_{2}\right) d_{c}\left(p_{1}\right),
\end{aligned}
$$$$
V_{e^{-} e^{+} \rightarrow \gamma \gamma}\left(k_{2}, k_{1} ; p_{2}, p_{1}\right)=\frac{e^{2} m}{2(2 \pi)^{3}} \times
$$$$
\times \delta\left(\boldsymbol{p}_{1}+\boldsymbol{p}_{2}-\boldsymbol{k}_{1}-\boldsymbol{k}_{2}\right)\left[v_{e^{-} e^{+} \rightarrow \gamma \gamma}^{\text {Feynman-like }}+v_{e^{-} e^{+} \rightarrow \gamma \gamma}^{\text {off-energy-shell }}\right],
$$$$
v_{e^{-} e^{+} \rightarrow \gamma \gamma}^{\text {Feynan-like }}=\frac{\bar{v}\left(p_{1}\right) \phi\left(k_{1}\right) \phi\left(k_{2}\right) u\left(p_{2}\right)}{\not \phi_{1}-\not \phi_{1}+m},
$$$$
v_{e^{-} e^{+} \rightarrow \gamma \gamma}^{\text {off-energy-shell }}=-\frac{1}{2}\left[\frac{\bar{v}\left(p_{1}\right) \phi\left(k_{1}\right) \phi\left(k_{2}\right) u\left(p_{2}\right)}{\not p_{1}-\not \phi_{1}+m}+\right.
$$$$
\left.+\frac{\bar{v}\left(p_{1}\right) \phi\left(k_{2}\right) \notin\left(k_{1}\right) u\left(p_{2}\right)}{\not p_{2}-\not p_{1}-m}\right] \text {. }
$$

\section{0}

The corresponding matrix element (quasipotential) is determined as

$$
\begin{aligned}
& \bar{V}_{e^{-} e^{+} \rightarrow \gamma \gamma}\left(k_{2}, k_{1} ; p_{2}, p_{1}\right)= \\
& =\left\langle c_{c}^{\dagger}\left(k_{2}\right) c_{c}^{\dagger}\left(k_{1}\right) \Omega\left|K_{e^{-} e^{+} \rightarrow \gamma \gamma}\right| b_{c}^{\dagger}\left(p_{2}\right) d_{c}^{\dagger}\left(p_{1}\right) \Omega\right\rangle= \\
& =-V_{e^{-} e^{+} \rightarrow \gamma \gamma}\left(k_{2}, k_{1} ; p_{2}, p_{1}\right)- \\
& -V_{e^{-} e^{+} \rightarrow \gamma \gamma}\left(k_{1}, k_{2} ; p_{2}, p_{1}\right)= \\
& =\frac{e^{2} m}{2(2 \pi)^{3}} \delta\left(\boldsymbol{p}_{1}+\boldsymbol{p}_{2}-\boldsymbol{k}_{1}-\boldsymbol{k}_{2}\right) \bar{v}_{e^{-} e^{+} \rightarrow \gamma \gamma}\left(k_{2}, k_{1} ; p_{2}, p_{1}\right),
\end{aligned}
$$

$\bar{v}_{e^{-} e^{+} \rightarrow \gamma \gamma}\left(k_{2}, k_{1} ; p_{2}, p_{1}\right)=\bar{v}\left(p_{1}\right) \phi\left(k_{1}\right) \times$

$$
\begin{aligned}
& \times \frac{1}{2}\left\{\frac{1}{\not p_{2}-\not \not_{2}-m}-\frac{1}{\not p_{1}-\not p_{1}+m}\right\} \not\left(k_{2}\right) u\left(p_{2}\right)+ \\
& +\bar{v}\left(p_{1}\right) \phi\left(k_{2}\right) \frac{1}{2}\left\{\frac{1}{\not p_{2}-\not p_{1}-m}-\frac{1}{\not p_{1}-\not p_{2}+m}\right\} \times
\end{aligned}
$$$$
\times \notin\left(k_{1}\right) u\left(p_{2}\right) \text {. }
$$

The Feynman-like propagators in the curly brackets of this expression appear after the summation of the corresponding noncovariant propagators which are illustrated on OFPT graphs in Fig. 6. For example, by adding contributions $(a)$ and $(b)$, we get

$$
\begin{aligned}
& \frac{m}{E_{\boldsymbol{k}_{1}-\boldsymbol{p}_{1}}}\left[\frac{P_{+}(q)}{E_{\boldsymbol{p}_{1}}-\omega_{\boldsymbol{k}_{1}}+E_{\boldsymbol{k}_{1}-\boldsymbol{p}_{1}}}+\frac{P_{-}\left(q_{-}\right)}{E_{\boldsymbol{p}_{1}}-\omega_{\boldsymbol{k}_{1}}-E_{\boldsymbol{k}_{1}-\boldsymbol{p}_{\mathbf{1}}}}\right]= \\
& =\frac{1}{\not p_{1}-\not \phi_{1}+m},
\end{aligned}
$$

with the four vector $q=\left(E_{\boldsymbol{k}_{1}-\boldsymbol{p}_{1}}, \boldsymbol{k}_{1}-\boldsymbol{p}_{1}\right)$ and the projection operators on the fermion positive (negative)-energy states $P_{ \pm}(q)=(q \pm m) / 2 m$.

According to [5], we introduce "left" $\left(s_{1}, u_{1}\right.$, and $\left.t_{1}\right)$ and "right" $\left(s_{2}, u_{2}\right.$, and $\left.t_{2}\right)$ Mandelstam vectors

$$
\begin{array}{lll}
s_{1}=p_{1}+k_{1}, & s_{2}=p_{2}+k_{2}, & u_{1}=p_{1}-k_{2}, \\
u_{2}=p_{2}-k_{1}, & t_{1}=p_{1}-k_{1}, & t_{2}=p_{2}-k_{2} .
\end{array}
$$

ISSN 2071-0194. Ukr. J. Phys. 2021. Vol. 66, No. 10 

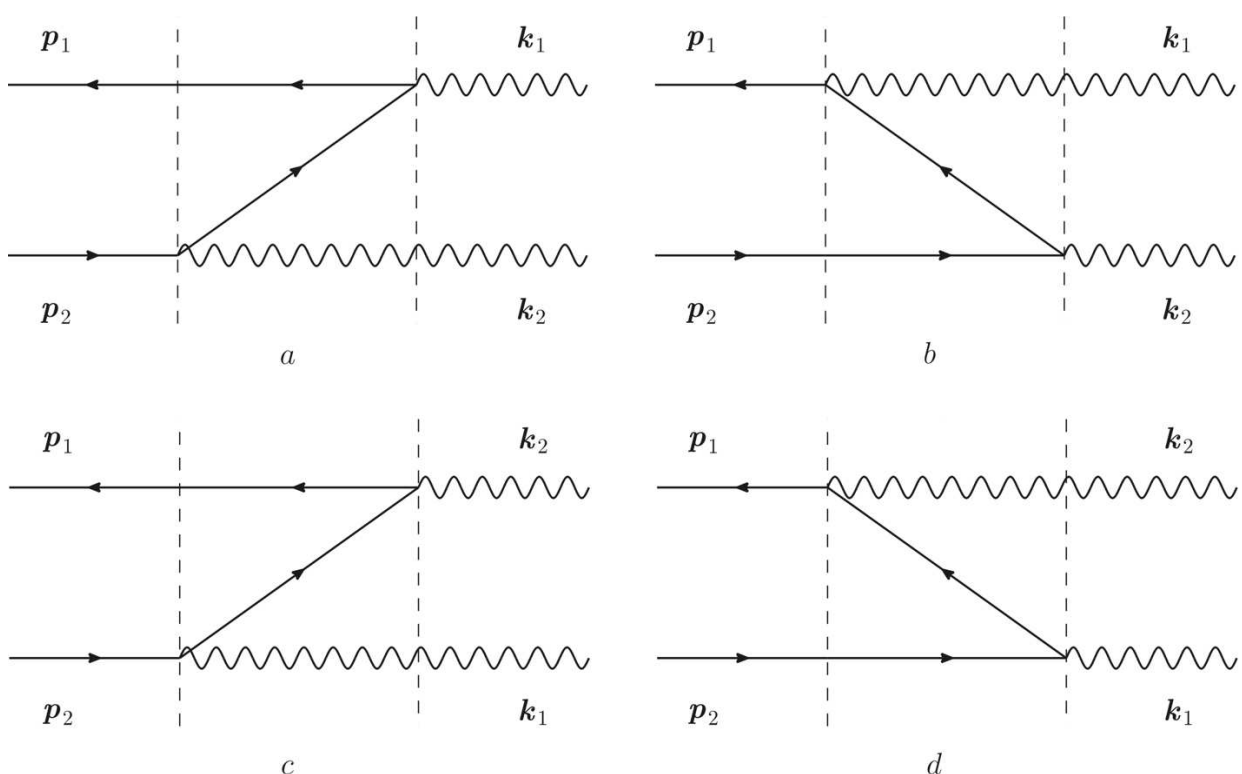

Fig. 6. The direct $(a, b)$ and exchange $(c, d)$ OFPT diagrams for interaction (46)

In these notations, expression (47) looks as

$\bar{v}_{e^{-} e^{+\gamma \gamma}}\left(k_{2}, k_{1} ; p_{2}, p_{1}\right)=$

$=\bar{v}\left(p_{1}\right) \phi\left(k_{1}\right) \frac{1}{2}\left\{\frac{1}{t_{2}-m}-\frac{1}{\not_{1}+m}\right\} \phi\left(k_{2}\right) u\left(p_{2}\right)+$

$+\bar{v}\left(p_{1}\right) \phi\left(k_{2}\right) \frac{1}{2}\left\{\frac{1}{\psi_{2}-m}-\frac{1}{\psi_{1}+m}\right\} \phi\left(k_{1}\right) u\left(p_{2}\right)$.

On the energy shell, $E_{\boldsymbol{p}_{1}}+E_{\boldsymbol{p}_{2}}=\omega_{\boldsymbol{k}_{1}}+\omega_{\boldsymbol{k}_{2}}$, and the expressions in the curly brackets are transformed into the Feynman propagators

$\frac{1}{2}\left\{\frac{1}{\not p_{2}-\not \not_{2}-m}-\frac{1}{\not \not_{1}-\not \phi_{1}+m}\right\} \rightarrow$

$\rightarrow \frac{1}{\not p_{2}-\not \not_{2}-m}$

$\frac{1}{2}\left\{\frac{1}{\not p_{2}-\not \phi_{1}-m}-\frac{1}{\not \not_{1}-\not \phi_{2}+m}\right\} \rightarrow$

$$
\rightarrow \frac{1}{\not p_{2}-\not \phi_{1}-m} \text {. }
$$

This occurs, when we evaluate the $S$-matrix for the $e^{+} e^{-}$-pair annihilation. The corresponding Feynman diagrams are displayed in Fig. 7.

\subsection{The interaction operator \\ for $e^{-} e^{+}$-pair production}

Operator (43) is not Hermitian, and its Hermitian conjugate describes the $e^{+} e^{-}$-pair production

$K_{\gamma \gamma \rightarrow e^{-} e^{+}}=\int \frac{d \boldsymbol{k}_{1}}{\omega_{\boldsymbol{k}_{1}}} \frac{d \boldsymbol{k}_{2}}{\omega_{\boldsymbol{k}_{2}}} \frac{d \boldsymbol{p}_{1}}{E_{\boldsymbol{p}_{1}}} \frac{d \boldsymbol{p}_{2}}{E_{\boldsymbol{p}_{2}}} \times$

ISSN 2071-0194. Ukr. J. Phys. 2021. Vol. 66, No. 10

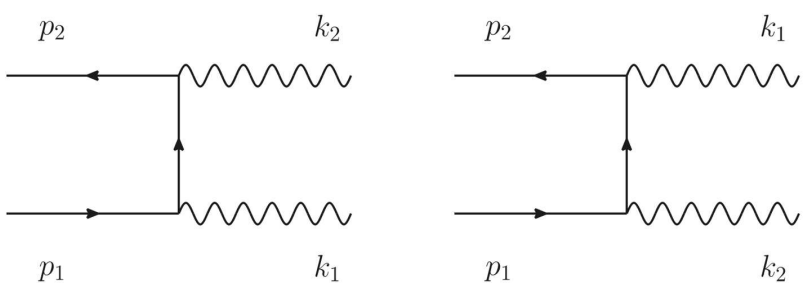

Fig. 7. The Feynman diagrams of the $e^{2}$-order for the annihilation process

$$
\begin{aligned}
& \times V_{\gamma \gamma \rightarrow e^{-} e^{+}}\left(p_{2}, p_{1} ; k_{2}, k_{1}\right) b_{c}^{\dagger}\left(p_{2}\right) \times \\
& \times d_{c}^{\dagger}\left(p_{1}\right) c_{c}\left(k_{2}\right) c_{c}\left(k_{1}\right), \\
& V_{\gamma \gamma \rightarrow e^{-} e^{+}}\left(p_{2}, p_{1} ; k_{2}, k_{1}\right)=\frac{e^{2} m}{2(2 \pi)^{3}} \times \\
& \times \delta\left(\boldsymbol{p}_{1}+\boldsymbol{p}_{2}-\boldsymbol{k}_{1}-\boldsymbol{k}_{2}\right)\left[v_{\gamma \gamma \rightarrow e^{-} e^{+}}^{\text {Feynman-like }}+v_{\gamma \gamma \rightarrow e^{-} e^{+}}^{\text {off-energy-shell }}\right] \\
& v_{\gamma \gamma \rightarrow e^{-} e^{+}}^{\text {Feynman-like }}=-\frac{\bar{u}\left(p_{2}\right) \phi\left(k_{1}\right) \phi\left(k_{2}\right) v\left(p_{1}\right)}{\not \phi_{1}-\not k_{1}+m} \\
& v_{\gamma \gamma \rightarrow e^{-} e^{+}}^{\text {off-energy-shell }}=\frac{1}{2}\left[\frac{\bar{u}\left(p_{2}\right) \phi\left(k_{1}\right) \phi\left(k_{2}\right) v\left(p_{1}\right)}{\not \phi_{1}-\not l_{1}+m}+\right. \\
& \left.+\frac{\bar{u}\left(p_{2}\right) \phi\left(k_{2}\right) \phi\left(k_{1}\right) v\left(p_{1}\right)}{\not \phi_{2}-\not k_{1}-m}\right] .
\end{aligned}
$$

To retain the hermiticity of the total Hamiltonian, operators (43) and (52) should be considered jointly. 


\subsection{The interaction operator for the Compton scattering}

The Compton scattering is described by the operator with a structure $b_{c}^{\dagger} c_{c}^{\dagger} b_{c} c_{c}$

$$
\begin{aligned}
& K_{\gamma e^{-} \rightarrow \gamma e^{-}}=\int \frac{d \boldsymbol{k}_{2}}{\omega_{\boldsymbol{k}_{2}}} \frac{d \boldsymbol{p}_{2}}{E_{\boldsymbol{p}_{2}}} \frac{d \boldsymbol{k}_{1}}{\omega_{\boldsymbol{k}_{1}}} \frac{d \boldsymbol{p}_{1}}{E_{\boldsymbol{p}_{1}}} \times \\
& \times V_{\gamma e^{-}}\left(p_{2}, k_{2} ; p_{1}, k_{1}\right) b_{c}^{\dagger}\left(p_{2}\right) c_{c}^{\dagger}\left(k_{2}\right) b_{c}\left(p_{1}\right) c_{c}\left(k_{1}\right), \\
& V_{\gamma e^{-}}\left(p_{2}, k_{2} ; p_{1}, k_{1}\right)=\frac{e^{2} m}{2(2 \pi)^{3}} \delta\left(\boldsymbol{p}_{1}+\boldsymbol{k}_{1}-\boldsymbol{p}_{2}-\boldsymbol{k}_{2}\right) \times \\
& \times\left[v_{\gamma e^{-}}^{\text {Feynman-like }}+v_{\gamma e^{-}}^{\text {off-energy-shell }}\right] \text {, } \\
& v_{\gamma e^{-}}^{\text {Feynman-like }}=\bar{u}\left(p_{2}\right)\left\{\phi\left(k_{2}\right) \frac{1}{\not p_{1}+\not \phi_{1}-m} \phi\left(k_{1}\right)+\right. \\
& \left.+\phi\left(k_{1}\right) \frac{1}{\not p_{1}-\not p_{2}-m} \phi\left(k_{2}\right)\right\} u\left(p_{1}\right) \text {, } \\
& v_{\gamma e^{-}}^{\text {off-energy-shell }}=\bar{u}\left(p_{2}\right) \phi\left(k_{2}\right) \times \\
& \times \frac{1}{2}\left\{\frac{1}{\not p_{2}+\not p_{2}-m}-\frac{1}{\not p_{1}+\not p_{1}-m}\right\} \phi\left(k_{1}\right) u\left(p_{1}\right)+ \\
& +\bar{u}\left(p_{2}\right) \phi\left(k_{1}\right) \frac{1}{2}\left\{\frac{1}{\not p_{2}-\not p_{1}-m}-\frac{1}{\not p_{1}-\not p_{2}-m}\right\} \times \\
& \times \notin\left(k_{2}\right) u\left(p_{1}\right) \text {. }
\end{aligned}
$$

The corresponding quasipotential is

$\bar{V}_{\gamma e^{-}}\left(p_{2}, k_{2} ; p_{1}, k_{1}\right)=$

$=\left\langle c_{c}^{\dagger}\left(k_{2}\right) b_{c}^{\dagger}\left(p_{2}\right)\left|K_{\gamma e^{-} \rightarrow \gamma e^{-}}\right| c_{c}^{\dagger}\left(k_{1}\right) b_{c}^{\dagger}\left(p_{1}\right)\right\rangle=$

$=V_{\gamma e^{-}}\left(p_{2}, k_{2} ; p_{1}, k_{1}\right)$.

For the interpretation of expression (58), we write an intermediate analytic result that leads to it:

$v_{\gamma e^{-}}\left(p_{2}, k_{2} ; p_{1}, k_{1}\right)=\frac{1}{2} \bar{u}\left(p_{2}\right) \times$

$\times\left\{\phi\left(k_{2}\right) P\left(p_{2}, k_{2} ; p_{1}, k_{1}\right) \phi\left(k_{1}\right)+\left(k_{2}, p_{2} \leftrightarrow k_{1}, p_{1}\right)\right\} u\left(p_{1}\right)$,

where

$$
\begin{aligned}
& P\left(p_{2}, k_{2} ; p_{1}, k_{1}\right)=\frac{m}{E_{\boldsymbol{p}_{1}+\boldsymbol{k}_{1}}}\left\{\frac{P_{+}\left(E_{\boldsymbol{p}_{1}+\boldsymbol{k}_{1}}, \boldsymbol{p}_{1}+\boldsymbol{k}_{1}\right)}{E_{\boldsymbol{p}_{1}}+\omega_{\boldsymbol{k}_{1}}-E_{\boldsymbol{p}_{1}+\boldsymbol{k}_{1}}}+\right. \\
& \left.+\frac{P_{-}\left(E_{\boldsymbol{p}_{1}+\boldsymbol{k}_{1}},-\boldsymbol{p}_{1}-\boldsymbol{k}_{1}\right)}{E_{\boldsymbol{p}_{1}}+\omega_{\boldsymbol{k}_{1}}+E_{\boldsymbol{p}_{1}+\boldsymbol{k}_{1}}}\right\}+ \\
& +\frac{m}{E_{\boldsymbol{p}_{2}-\boldsymbol{k}_{1}}}\left\{\frac{P_{+}\left(E_{\boldsymbol{p}_{2}-\boldsymbol{k}_{1}}, \boldsymbol{p}_{2}-\boldsymbol{k}_{1}\right)}{E_{\boldsymbol{p}_{2}}-\omega_{\boldsymbol{k}_{1}}-E_{\boldsymbol{p}_{2}-\boldsymbol{k}_{1}}}+\right.
\end{aligned}
$$

$\left.+\frac{P_{-}\left(E_{\boldsymbol{p}_{2}-\boldsymbol{k}_{1}},-\boldsymbol{p}_{2}+\boldsymbol{k}_{1}\right)}{E_{\boldsymbol{p}_{2}}-\omega_{\boldsymbol{k}_{1}}+E_{\boldsymbol{p}_{2}-\boldsymbol{k}_{1}}}\right\}$

Each contribution to the r.h.s. of Eq. (60) can be represented by the graphs in Fig. 8. Graphs $(a)$ and (c) correspond to the two terms in the first curly brackets:

$\left.D_{a}(E)\right|_{E=E_{\boldsymbol{p}_{1}}+\omega_{\boldsymbol{k}_{1}}} \equiv\left(E-E_{\boldsymbol{p}_{1}+\boldsymbol{k}_{1}}\right)_{E=E_{\boldsymbol{p}_{1}}+\omega_{\boldsymbol{k}_{1}}}^{-1}=$ $=\left(E_{\boldsymbol{p}_{1}}+\omega_{\boldsymbol{k}_{1}}-E_{\boldsymbol{p}_{1}+\boldsymbol{k}_{1}}\right)^{-1}$,

$\left.D_{c}(E)\right|_{E=E_{\boldsymbol{p}_{2}}+\omega_{\boldsymbol{k}_{2}}} \equiv$

$\equiv\left(E-E_{\boldsymbol{p}_{1}}-E_{\boldsymbol{p}_{2}}-\omega_{\boldsymbol{k}_{1}}-\omega_{\boldsymbol{k}_{2}}-E_{\boldsymbol{p}_{1}+\boldsymbol{k}_{1}}\right)_{E=E_{\boldsymbol{p}_{2}}+\omega_{\boldsymbol{k}_{2}}}^{-1}=$ $=-\left(E_{\boldsymbol{p}_{1}}+\omega_{\boldsymbol{k}_{1}}+E_{\boldsymbol{p}_{1}+\boldsymbol{k}_{1}}\right)^{-1}$,

while graphs $(b)$ and $(d)$ are associated with the two ones in the second curly brackets.

In terms of the Mandelstam variables (49), expression (59) takes the form

$v_{\gamma e^{-}}\left(p_{2}, k_{2} ; p_{1}, k_{1}\right)=\bar{u}\left(p_{2}\right) \phi\left(k_{2}\right) \times$

$\times\left\{\frac{1}{2} \frac{1}{\$_{1}-m}+\frac{1}{2} \frac{1}{\$_{2}-m}\right\} \phi\left(k_{1}\right) u\left(p_{1}\right)+$

$+\bar{u}\left(p_{2}\right) \phi\left(k_{1}\right)\left\{\frac{1}{2} \frac{1}{\psi_{1}-m}+\frac{1}{2} \frac{1}{\psi_{2}-m}\right\} \times$

$\times \notin\left(k_{2}\right) u\left(p_{1}\right)$.

On the energy shell, $E_{\boldsymbol{p}_{1}}+\omega_{\boldsymbol{k}_{1}}=E_{\boldsymbol{p}_{2}}+\omega_{\boldsymbol{k}_{2}}$, where there is no difference between the left and right Mandelstam vectors, this equation reduces to

$\left.v_{e^{-} \gamma}\left(p_{2}, k_{2} ; p_{1}, k_{1}\right)\right|_{E_{\boldsymbol{p}_{1}}+\omega_{\boldsymbol{k}_{1}}=E_{\boldsymbol{p}_{2}}+\omega_{\boldsymbol{k}_{2}}}=$

$=\frac{\bar{u}\left(p_{2}\right) \phi\left(k_{2}\right) \phi\left(k_{1}\right) u\left(p_{1}\right)}{\$-m}+\frac{\bar{u}\left(p_{2}\right) \phi\left(k_{1}\right) \phi\left(k_{2}\right) u\left(p_{1}\right)}{\psi-m}$,

which coincides up to a numerical factor with the expressions, obtained with help of the Feynman rules from the diagrams in Fig. 9.

One can replace the Dirac spinors $(u \rightarrow v)$ in interactions (55) and (19) to obtain expressions for the corresponding processes with positrons.

In Ref. [19], there are the calculations of the $e^{-} e^{-}, e^{-} e^{+}$scattering and $e^{-} e^{+}$annihilation cross sections with the new interaction operators shown above. They reproduce the results obtained using the well-known formulas by Møller and Bhabha (see, e.g., [20]).

ISSN 2071-0194. Ukr. J. Phys. 2021. Vol. 66, No. 10 

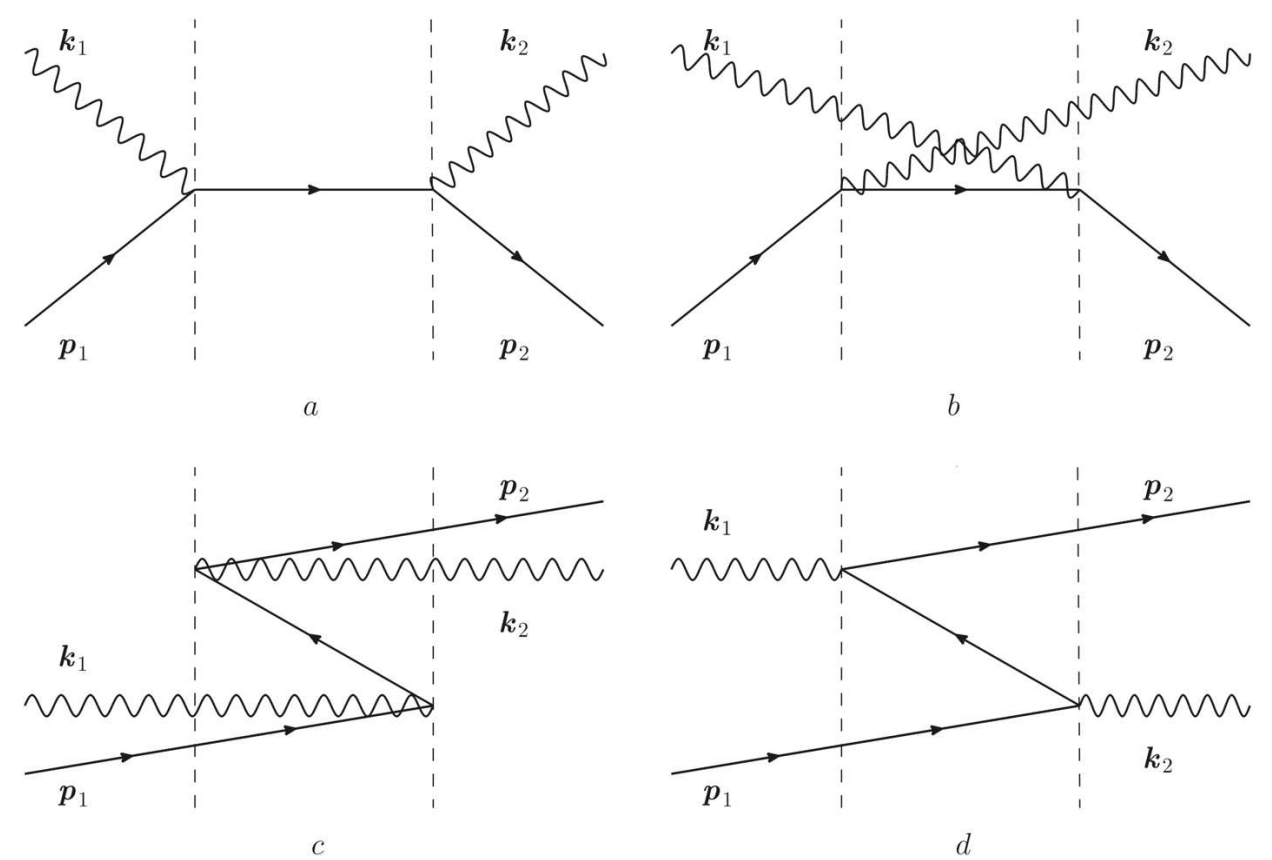

Fig. 8. OFPT diagrams for different contributions to interaction (58)

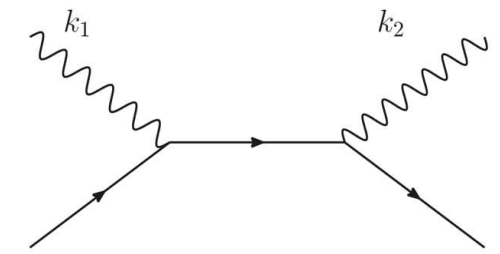

$p_{1}$

$p_{2}$

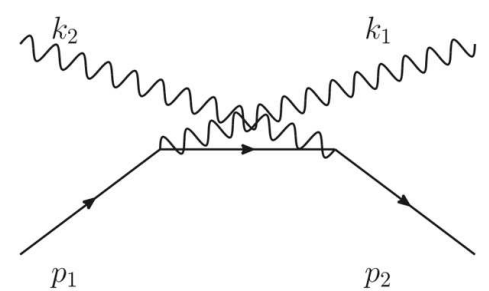

Fig. 9. Feynman diagrams for the Compton effect in the second order

The corresponding cross-sections in the $e^{2}$-order can be obtained from the interaction operator (19) using the Born approximation for the $T$-matrix

$\delta\left(\boldsymbol{p}_{f}-\boldsymbol{p}_{i}\right) T_{f i} \approx\left\langle f\left|K_{I}\right| i\right\rangle$,

where $\boldsymbol{p}_{i}\left(\boldsymbol{p}_{f}\right)$ is the total momentum in the initial (final) state. For example, for the $e^{-} e^{-}$-scattering, one has

$$
\begin{aligned}
& |i\rangle=\frac{1}{\sqrt{E_{\boldsymbol{p}_{1}} E_{\boldsymbol{p}_{\mathbf{2}}}}} b_{c}^{\dagger}\left(p_{1}\right) b_{c}^{\dagger}\left(p_{2}\right)|\Omega\rangle, \\
& |f\rangle=\frac{1}{\sqrt{E_{\boldsymbol{p}_{1}^{\prime}} E_{\boldsymbol{p}_{2}^{\prime}}}} b_{c}^{\dagger}\left(p_{1}^{\prime}\right) b_{c}^{\dagger}\left(p_{2}^{\prime}\right)|\Omega\rangle,
\end{aligned}
$$

which gives

$\left\langle f\left|K_{I}\right| i\right\rangle=\left\langle f\left|K_{e^{-} e^{\rightarrow} e^{-} e^{-}}\right| i\right\rangle=$
$=\bar{V}_{e^{-} e^{-}}\left(p_{1}^{\prime}, p_{2}^{\prime} ; p_{1}, p_{2}\right)$

with the quasipotential $\bar{V}_{e^{-} e^{-}}$defined in Eq. (25). The cross-section is calculated using the following expression (see Eq. (3.137) in Ref. $[21])$ :

$d \sigma=\int d \boldsymbol{p}_{1}^{\prime} d \boldsymbol{p}_{2}^{\prime} \delta\left(E_{\boldsymbol{p}_{1}^{\prime}}+E_{\boldsymbol{p}_{2}^{\prime}}-E_{\boldsymbol{p}_{1}}-E_{\boldsymbol{p}_{2}}\right) \times$

$\times \delta\left(\boldsymbol{p}_{1}^{\prime}+\boldsymbol{p}_{2}^{\prime}-\boldsymbol{p}_{1}-\boldsymbol{p}_{2}\right) \frac{(2 \pi)^{4}\left|T_{f i}\right|^{2}}{v_{\mathrm{rel}}}$,

where $v_{\text {rel }}$ is the relative speed of electrons in the initial state. Here, we encounter with the energy-shell condition $E_{\boldsymbol{p}_{1}^{\prime}}+E_{\boldsymbol{p}_{2}^{\prime}}=E_{\boldsymbol{p}_{1}}+E_{\boldsymbol{p}_{2}}$. Therefore, in the quasipotential $\bar{V}_{e^{-} e^{-}}$, the "off-energy-shell" contribution (27) vanishes. For an unpolarized initial beam 
and when the final polarization is not observed, the differential cross-section in the center-mass system (c.m.s.) is given by

$\frac{d \sigma}{d \Omega}=\frac{(2 \pi)^{4} E^{2}}{16} \sum_{\mu_{1}^{\prime}, \mu_{2}^{\prime}, \mu_{1}, \mu_{2}}\left|T_{f i}\right|^{2}$,

where $E$ is the c.m.s. energy of the pair of electrons. The $T$-matrix is

$T_{f i}=\frac{e^{2} m^{2}}{(2 \pi)^{3} E^{2}}\left(\frac{\bar{u}\left(p_{-}^{\prime} \mu_{2}^{\prime}\right) \gamma^{\mu} u\left(p \mu_{1}\right) \bar{u}\left(p^{\prime} \mu_{1}^{\prime}\right) \gamma_{\mu} u\left(p_{-} \mu_{2}\right)}{\left(p^{\prime}-p_{-}\right)^{2}}-\right.$

$\left.-\frac{\bar{u}\left(p^{\prime} \mu_{1}^{\prime}\right) \gamma^{\mu} u\left(p \mu_{1}\right) \bar{u}\left(p_{-}^{\prime} \mu_{2}^{\prime}\right) \gamma_{\mu} u\left(p_{-} \mu_{2}\right)}{\left(p^{\prime}-p\right)^{2}}\right)$

with $p=(E, \boldsymbol{p}), p_{-}=(E,-\boldsymbol{p})$ and $|\boldsymbol{p}|=\sqrt{E^{2}-m^{2}}$. After summations over polarizations and using the formulas for Dirac traces, we obtain the Møller formula

$$
\begin{aligned}
& \frac{d \sigma}{d \Omega}=\frac{\alpha^{2}\left(2 E^{2}-m^{2}\right)^{2}}{4 E^{2}}\left(\frac{4}{\sin ^{2} \theta}-\frac{3}{\sin ^{2} \theta}+\right. \\
& \left.+\frac{\left(E^{2}-m^{2}\right)^{2}}{\left(2 E^{2}-m^{2}\right)^{2}}\left(1+\frac{4}{\sin ^{2} \theta}\right)\right) .
\end{aligned}
$$

The Bhabha formula for the $e^{-} e^{+}$-scattering and the Klein-Nishina one for the Compton scattering are obtained in the same way by using the appropriate expressions for the corresponding initial and final states in Eq. (64).

N.B. At this point, we would like to note that such a transition to the CPR calculations of the corresponding $S$-matrix elements can be simplified with the help of the equivalence theorem [22]. It has turned out that the $S$ operators that are determined by the time evolution from a distant past to a distant future, for the two decompositions $H=H(\alpha)=H_{F}+H_{I}$ and $H=K\left(\alpha_{c}\right)=K_{F}+K_{I}$, being sandwiched between the bare states and the corresponding clothed ones are equal to each other. Such a coincidence becomes possible owing to a certain isomorphism between the $\alpha_{c}$ algebra and the $\alpha$ algebra, once the UCTs $W_{D}(t) \equiv \exp \left(i K_{F} t\right) W \exp \left(-i K_{F} t\right)$ obey the asymptotic condition $W_{D}( \pm \infty)=1$ (cf., analogous considerations on p. 114 of Ref. [6] for the NNscattering amplitudes).

\section{Conclusions}

Our consideration gives one more application of a well-forgotten concept on the clothed particles in

\section{4}

quantum field theory, put forward by Greenberg and Schweber [1]. We have seen that our approach leads to the new Hermitian and energy-independent interactions between clothed particles including the offenergy-shell and recoil effects (the latter in all orders of the $v^{2} / c^{2}$-expansion). These interactions have been verified on the energy shell, viz., they reproduce the well-known formulas for the cross-sections of elementary processes in the standard QED. Moreover, our results can be used for a more detailed study of the processes in which the off-energy-shell effects play an essential role. It is the case of the treatment of positronium properties, the simplest bound state in QED, and other processes involving bound states, e.g., the electron-positronium scattering, etc. Our explorations in this direction are underway.

1. O. Greenberg, S. Schweber. Clothed particle operators in simple models of quantum field theory. Nuovo Cim. 8, 378 (1958).

2. A. Shebeko, M. Shirokov. Clothing procedure in relativistic quantum field theory and its applications to description of electromagnetic interactions with nuclei (bound systems). Progr. Part. Nucl. Phys. 44, 75 (2000).

3. A. Shebeko, M. Shirokov. Unitary transformations in quantum field theory and bound states. Phys. Part. Nucl. 32, 15 (2001) [arXiv: nucl-th/0102037].

4. V. Korda, A. Shebeko. Clothed particles representation in quantum field theory: mass renormalization. Phys. Rev. D 70, 085011 (2004).

5. V. Korda, L. Canton, A. Shebeko. Relativistic interactions for the meson-two-nucleon system in the clothed-particle unitary representation. Ann. Phys. 322, 736 (2007).

6. I. Dubovyk, A. Shebeko. The method of unitary clothing transformations in the theory of nucleon-nucleon scattering. Few-Body Syst. 48, 109 (2010).

7. E. Dubovyk, A. Shebeko. Low-energy parameters of the nucleon-nucleon scattering and deuteron properties, electromagnetic interactions with bound systems. Few-Body Syst. 54, 1513 (2013).

8. A. Shebeko. The method of unitary clothing transformations in relativistic quantum field theory: Recent applications for the description of nucleon-nucleon scattering and deuteron properties. Few-Body Syst. 54, 2271 (2013).

9. H. Kamada, A. Shebeko, A. Arslanaliev. Triton binding energy of Kharkov potential. Few-Body Syst. 58, 70 (2017).

10. A. Shebeko. Clothed particles in mesodynamics, quantum electrodynamics and other field models. PoS Baldin ISHEPP XXII 225, 027 (2015).

11. A. Shebeko. Clothed particles in quantum electrodynamics and quantum chromodynamics. EPJ Web Conf. 113, 03014 (2016).

12. A. Arslanaliev, Y. Kostylenko, A. Shebeko. QED in the clothed-particle representation. In: Recent Progress in

ISSN 2071-0194. Ukr. J. Phys. 2021. Vol. 66, No. 10 
Few-Body Physics. Edited by N. Orr et al. (Springer, 2020), p. 41.

13. Y. Kostylenko, A. Arslanaliev, A. Shebeko. QED in the clothed-particle representation: A fresh look at positronium properties treatment. SciPost Phys. Proc. 3, 45 (2020).

14. S. Weinberg. The Quantum Theory of Fields (Cambridge University Press, 1995), Vol.1.

15. A. Shebeko, P. Frolov. A possible way for constructing generators of the Poincaré group in quantum field theory. FewBody Syst. 52, 125 (2012).

16. E. Stefanovich. Relativistic quantum dynamics: A nontraditional perspective on space, time, particles, fields, and action-at-a-distance. Ann. Phys. 322 (2007) [arXiv: physics/0504062].

17. S. Schweber. An Introduction to Relativistic Quantum Field Theory (Row, Peterson \& Co., 1961) [ISBN: 9780486442280].

18. A. Arslanaliev. MSc thesis. The Method of Unitary Clothing Transformations: Application in Quantum Electrodynamics (V.N. Karazin National University, 2017).

19. Y. Kostylenko. MSc thesis. The Application of the Method of Unitary Clothing Transformations in the Description of Positronium Properties (V.N. Karazin National University, 2019).

20. H. Kleinert. Particles and Quantum Fields (Freie Universität, 2016).

21. M. Goldberger, K. Watson. Collision Theory (Wiley, 1967).
22. A. Shebeko. The S-matrix in the method of unitary clothing transformations. Nucl. Phys. A 737, 252 (2004).

Received 29.03.21

А.М. Арсланалієв, Я.О. Костиленко, О.В. Шебеко

НОВЕ СІМЕЙСТВО ВЗАЄМОДІЙ МІЖ ОДЯГНЕНИМИ ЧАСТИНКАМИ В КЕД

Метод унітарних одягаючих перетворень (УОП) був застосований у квантовій електродинаміці (КЕД) за допомогою зображення одягнених частинок (ЗОЧ). У рамках ЗОЧ гамільтоніан для взаємодіючих електромагнітних та електрон-позитронних полів набуває форми, в якій оператори взаємодії, відповідальні за такі двочастинкові процеси, як $e^{-} e^{-} \rightarrow e^{-} e^{-}, e^{+} e^{+} \rightarrow e^{+} e^{+}, e^{-} e^{+} \rightarrow e^{-} e^{+}, e^{-} e^{+} \rightarrow \gamma \gamma$, $\gamma \gamma \rightarrow e^{-} e^{+}, \gamma e^{-} \rightarrow \gamma e^{-}$та $\gamma e^{+} \rightarrow \gamma e^{+}$, отримуються на одній і тій самій фізичній основі. Ці нові взаємодії включають ефекти поза енергетичною поверхнею (off-energy-shell effects) та ефекти віддачі (останні без будь-якого розкладання в ряд по $\left.(v / c)^{2}\right)$, а їхні матричні елементи на енергетичній поверхні відтворюють добре відомі результати, отримані в рамках теорії збурень на основі ряду Дайсона для $S$ матриці (зокрема, формула Меллера для $e^{-} e^{-}$-розсіяння, формула Баба для $e^{-} e^{+}$-розсіяння та формула КляйнаНішини для комптонівського розсіювання).

Kлючов $i$ слова: унітарні одягаючі перетворення, квантова електродинаміка, одягнені частинки, ефекти поза енергетичною поверхнею. 